\title{
3D Analysis of the Alignment of the Lower Extremity in High Tibial Osteotomy
}

\author{
Hideo Kawakami ${ }^{1}$, Nobuhiko Sugano ${ }^{2}$,Takashi Nagaoka ${ }^{5}$, \\ Keisuke Hagio ${ }^{1}$, Kazuo Yonenobu ${ }^{3}$, Hideki Yoshikawa ${ }^{2}$, \\ Takahiro Ochi $^{1}$, Asaki Hattori ${ }^{4}$, and Naoki Suzuki ${ }^{4}$ \\ ${ }^{1}$ Department of Computer Integrated Orthopaedics, Osaka Univ. \\ Graduate School of Medicine, 2-2 Yamadaoka, Suita-shi, 565-0871,Osaka, Japan \\ hkawakami-osk@umin.ac.jp \\ ${ }^{2}$ Department of Orthopaedic Surgery, Osaka Univ. \\ Graduate School of Medicine, 2-2 Yamadaoka, Suita-shi, 565-0871,Osaka, Japan \\ ${ }^{3}$ Department of Orthopaedic Surgery, Osaka-Minami National Hospital, \\ 2-1 Kidohigashimachi, Kawachinagano-shi, 586-0008, Osaka, Japan \\ ${ }^{4}$ Institute for High Dimensional Medical Imaging, Jikei Univ.School of Medicine, \\ 4-11-1 Izumi Honcho, Komae-shi, Tokyo, Japan \\ ${ }^{5}$ Graduate School of Science and Engineering, Waseda University, \\ 3-4-1 Okubo, Shinjuku-ku, Tokyo, Japan
}

\begin{abstract}
The alignment of the lower extremities after high tibial osteotomy (HTO) varies widely on the radiographs. It is difficult to assess the rotation change on the radiographs. We developed computer software to calculate the alignment of the lower extremities and to simulate HTO. The purpose of this study is to compare and evaluate the variance in the femoro-tibial angle (FTA) and hip-knee-ankle angle (HKA) in relation to rotational shift of the lower extremity on radiographs and to clarify how the rotational shift affects the FTA and HKA by using three-dimensional (3D) CT simulation software for HTO. The mean absolute rotation angle of the lower extremity position on radiographs was 7.8 degrees, ranging from an external rotation of 8 degrees to an internal rotation of 14 degrees. Within the same range of rotation, the mean change in the FTA was 3.7 degrees, the mean change in the HKA was 1.5 degrees.
\end{abstract}

\section{Introduction}

High tibial osteotomy (HTO) is a treatment option for medial osteoarthritis of the knee. The goal of this operation is to reduce the abnormal loading stresses on the knee joint through realignment of the lower extremity by osteotomy of the proximal tibia. Researchers have recognized the importance of precise realignment after HTO, and it has been reported that poor alignment yields poor results overall ${ }^{35)(2)}$. However, alignment after HTO varies widely despite improvements in surgical techniques and instruments ${ }^{1667) 910)}$. Imprecise preoperative radiographic measurements and operative rotational shift of the osteotomy plane may be the factors which causes inconstancy of the postoperative alignment. The femoro-tibial angle (FTA) and hip-knee-ankle angle (HKA) are often used to estimate the axial alignment ${ }^{1) 11}$. FTA is an angle formed by the femoral axis and the tibial axis. HKA is an angle formed by the line from the 
center of the femoral head to the center of the knee joint and the line from the center of the knee joint to the center of the ankle joint. Preoperative planning of realignment involves measuring full-length weight-bearing radiographs of the lower extremity, but full-length radiographs may lack standardization in the positioning of the subjects and may be distorted by parallax. It is quite difficult to estimate axial alignment error due to rotational shift of the lower extremity on plain radiographs. In a closed wedge type of HTO, the proximal tibia is cut in a plane parallel to the knee joint surface, a wedged bone is resected from the proximal tibia on the lateral side, and each surface of the fragments is reattached. This procedure may lead to a shift in rotation between the proximal tibia and the distal tibia, and such a rotational shift causes a change in the axial alignment of the lower extremity. The purpose of this study is to evaluate and compare the changes of FTA and HKA in relation to rotational shift of the lower extremity on radiographs and to clarify how the rotational shift in osteotomy affects the FTA and HKA by using 3D CT simulation software for HTO.

\section{Materials and Methods}

22 knees of 13 patients with medial osteoarthritis of the knee were subjects of this study. There were 4 men and 9 women and the age ranged from 31 to 84 years (mean 64 years). An anteroposterior radiograph of the lower extremity was taken by positioning the extremity as much as possible so that the patellar was facing forward, and CT images were acquired from the proximal end of the femur to the distal end of the tibia by positioning with full extension of the knee joint and 90-degree extension of the ankle joint in supine position. 3D surface models of the femur, tibia and patellar were reconstructed from CT images by surface rendering. Six landmark points were plotted on the 3D surface models (Fig.1).

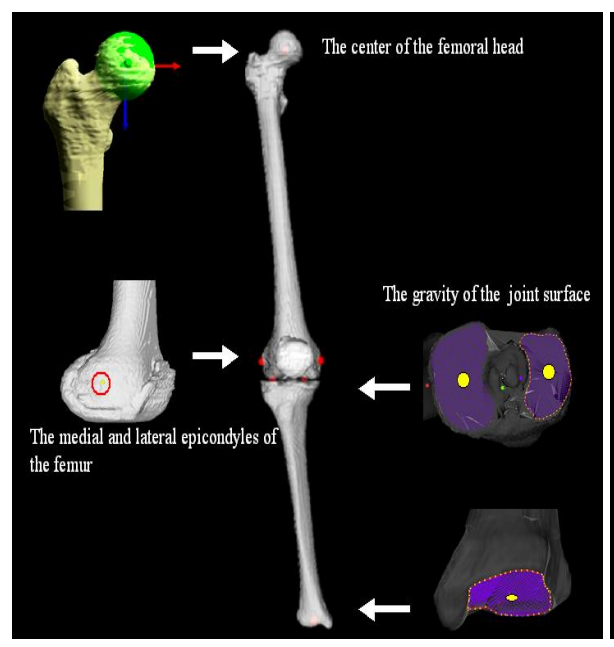

Fig. 1. Landmark points on the 3D surface model

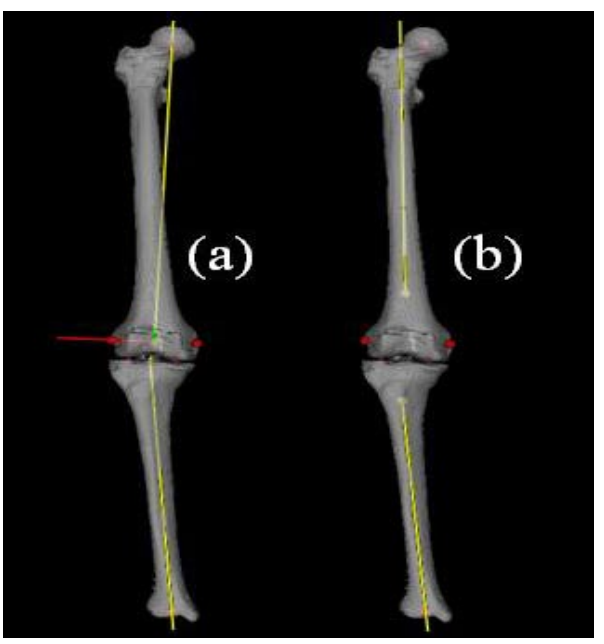

Fig. 2. Axis of the lower extremities (a) Mechanical axis for measuring HKA (b) Anatomical axis for measuring FTA 


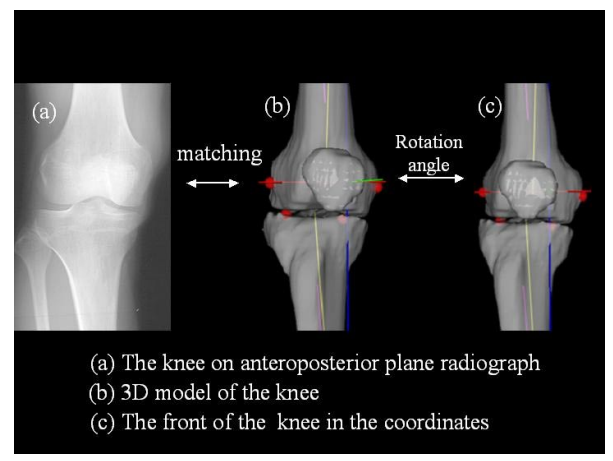

Fig. 3. The measurement of the axial rotation

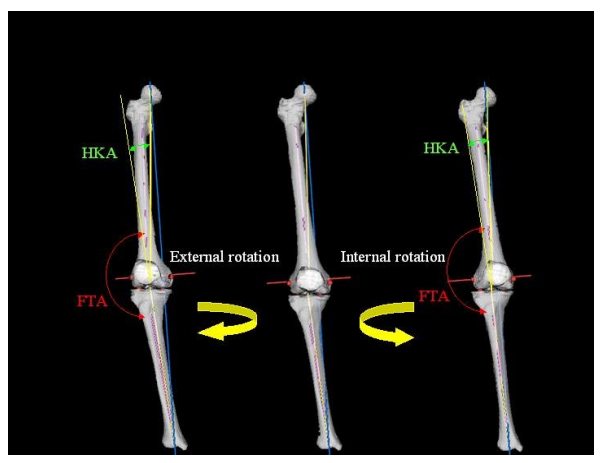

Fig. 4. The measurement of the alignment (FTA,HKA)

The first point was the center of the femoral head, which was determined by the least-squares method; the second and third points were the lateral and medial epicondyles of the femur, which were determined by selecting the most distant points among the 1-100 points around both; the fourth and fifth points represented the medial and lateral joint surface of the proximal tibia, and they were determined by selecting the centers of gravity of multiple points on each joint surface; the last point represented the joint surface center of the distal tibia, which was determined by the same method as the fourth and fifth points. Based on these points, the following coordinates were defined. In the coordinates to define the front of the lower extremity, the Z-axis is a line through the center of the femoral head and the center of gravity of the distal tibia joint surface, and the $\mathrm{X}$-axis is a line through the medial and lateral epicondyles of the femur. In the anatomical axis for measuring the FTA, the femoral axis is the line through the center of the femoral section with $15 \%$ proximal side of the femoral length and the center of the femoral section with $15 \%$ distal side of the femoral length, and the tibial axis is the line through the center of the tibia section with $15 \%$ proximal side of the tibial length and the center of the tibial section with $15 \%$ distal side of the tibial length (Fig.2-a). In the mechanical axis for measuring the HKA, the femoral axis is the line from the center of the femoral head to the mid-point of the medial and lateral epicondyle, and the tibial axis is the line from the center of gravity of the proximal tibia joint surface to the center of gravity of the distal tibia joint surface (Fig.2-b). We developed computer software that calculates the FTA and HKA on a $2 \mathrm{D}$ projection from the $3 \mathrm{D}$ bone models, and also developed a bone-cutting program which can cut the bone models in any plane and remove the wedge bone and reattach the surfaces for the operative simulation of HTO. To measure the axial rotation of the lower extremity on anteroposterior plane radiographs, position of the patellar and femur on the 3D surface model were matched to those of the patellar and femur on radiographs (Fig.3). The maximum external rotation and the maximum internal rotation of the lower extremity on the radiographs were picked up in all subjects, and the 3D surface model was rotated between assumed maximum internal rotation and external rotation, the FTA and HKA were calculated on the projection of the $3 \mathrm{D}$ models (Fig.4). Differences of HKA and FTA between the two assumed positions of the lower extremity were compared statistically. Using the operative simulation program, the FTA and HKA were measured in the hypothetical case of internal and external 10- 
degrees rotation error in cutting the proximal tibia and another hypothetical case of internal and external 10- degrees rotation error in reattaching proximal cutting surface to distal cutting surface(Fig.5). Similarly, differences of the FTA and HKA between the two types of HTO simulations were compared statistically.

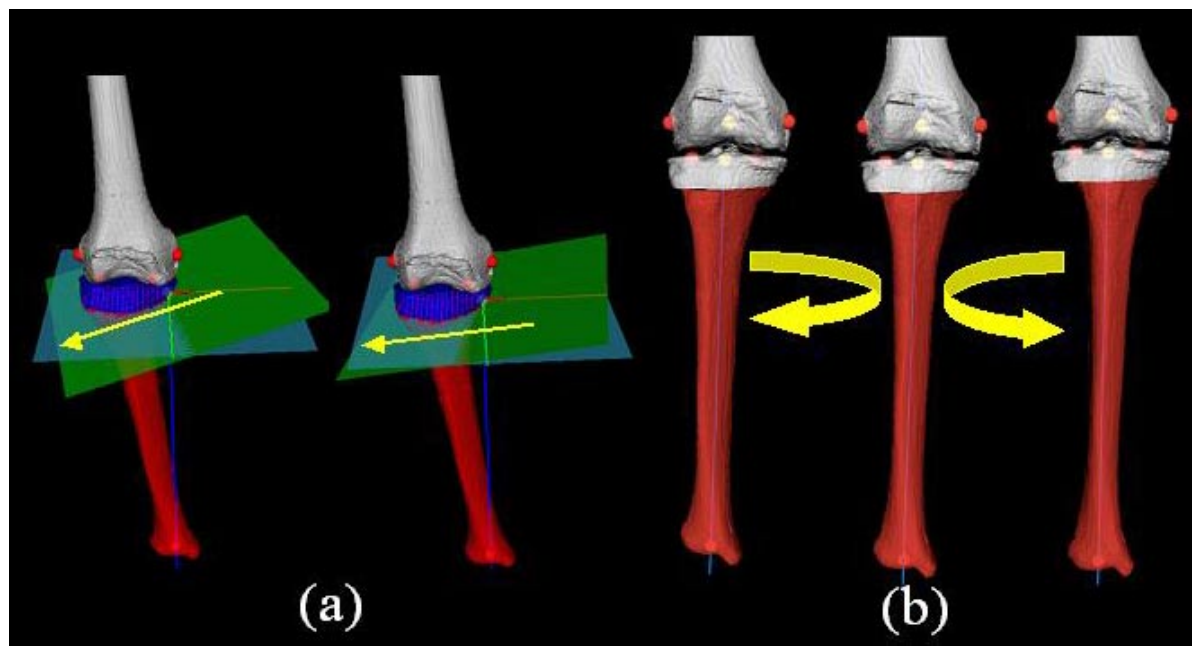

Fig. 5. Operative simulation of HTO

(a) Rotational error in cutting the proximal tibia (b) Rotational error in reattaching the bone cutting surface

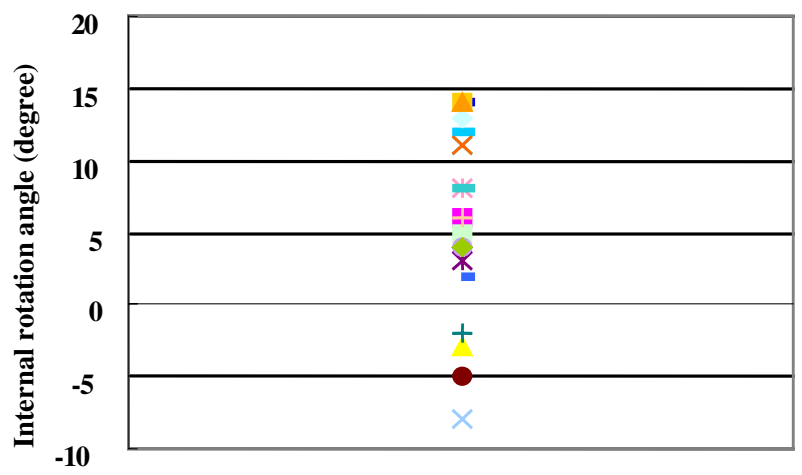

Fig. 6. Rotation angle of lower extremities on the radiographs $(\mathrm{N}=22)$

\section{Results}

The mean absolute rotation angle of the lower extremity position on radiographs was 7.8 degrees $(\mathrm{N}=22)$, ranging from an external rotation of 8 degrees to an internal rotation of 14 degrees (Fig.6). Within this range of rotation, the FTA tended to decrease with internal rotation, and the HKA tended to increase with internal rotation (Fig.7). Within the same range of rotation, the mean change in the FTA was 3.7 degrees, the mean change in the HKA was 1.5 degrees, and the differences of the FTA and HKA were significant $(\mathrm{P}<0.05)($ Fig.8). 

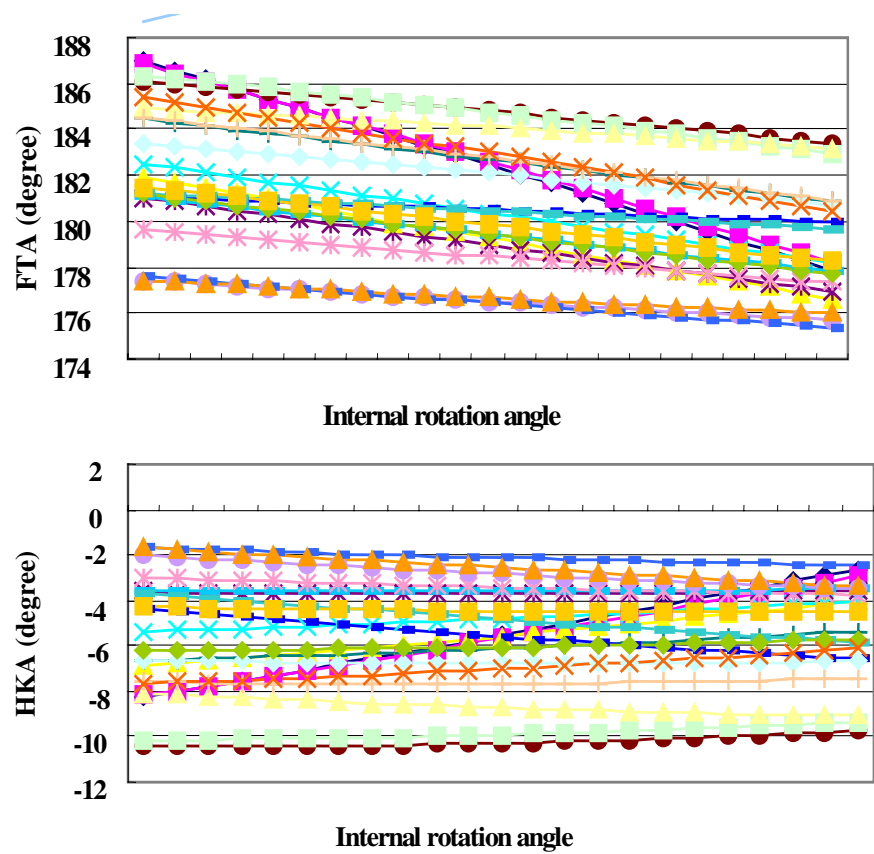

Fig. 7. Alignment of the lower extremities ranging from an external rotation of 8 degree to an internal rotation of 14 degrees $(\mathrm{N}=22)$

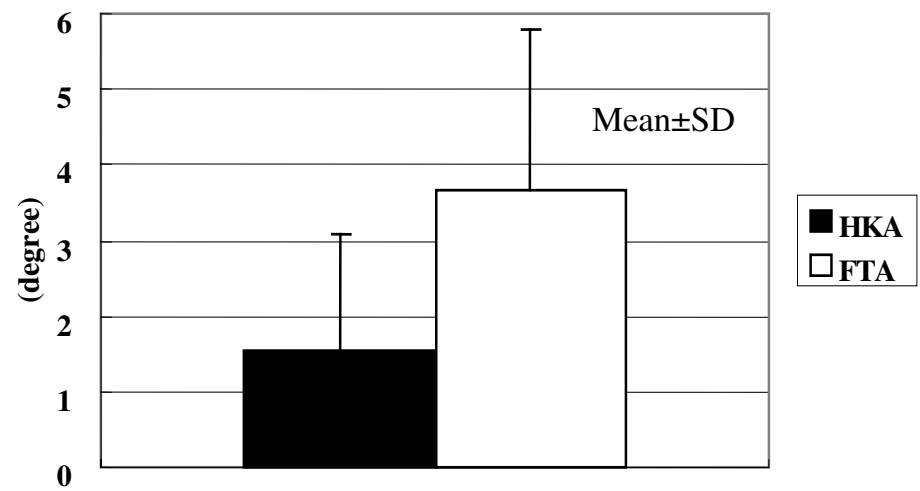

Fig. 8. Change in the HKA and FTA ranging from an external rotation of 8 degrees to an internal rotation of 14 degrees $(\mathrm{N}=22)$

When the rotational shift was assumed to be an internal and external rotation in the range of 10 degrees, the change in the axial alignment during the simulation of a rotational error in cutting the proximal tibia involved a mean of 0.4 degrees in the FTA and a mean of 0.3 degrees in the HKA, and the change in the axial alignment during the simulation of a rotational error in reattaching the proximal and distal bone surfaces involved a mean of 3.4 degrees in the FTA and a mean of 2.1 degrees in the HKA. The FTA tended to decrease with internal rotation of the distal tibia reattach- 
ment, and the HKA tended to increase with internal rotation of the distal tibia reattachment. Differences of the FTA and HKA during the simulation of a rotational error in cutting the proximal tibia were significant $(\mathrm{P}<0.05)$. Differences of the FTA and HKA during the simulation of a rotational error in reattaching the proximal bone cutting surface to the distal bone surface were significant $(\mathrm{P}<0.05)($ Fig.9).

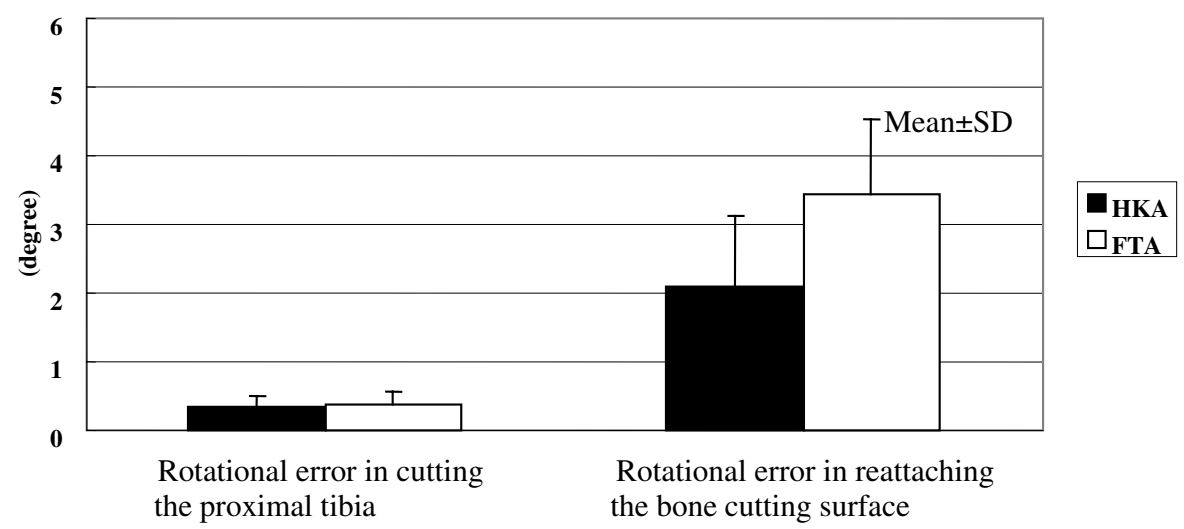

Fig. 9. Change in the HKA and FTA of HTO simulation $(\mathrm{N}=22)$

\section{Discussion}

The alignment of the lower extremities was calculated on the $2 \mathrm{D}$ radiographs, so that we could not evaluate the influence of rotation. In a previous study by Wright et al, lower extremities axial rotation of $10^{\circ}$ internally and externally did not have a statistically significant effect on FTA in radiographs of amputated lower extremities ${ }^{14)}$. By Swanson et al, lower extremities axial rotation of $10^{\circ}$ internally and externally have a statistically significant effect on FTA in radiographs of Sawbone extremities model with severe valgus or varus deformity ${ }^{13)}$. By Krackow ${ }^{8)}$ et al, the effect of flexion and rotation on varous and valgus deformity was analyzed by calculating the assuming leg deformity. Their results were different each other and materials of them were not patient with medial osteoarthritis of the knee. In this study the axial alignment of the patients with medial osteoarthritis of the knee was calculated and analysis, so it was useful on clinical analysis.

In previous study of a computer approach to HTO by Chao et al, 2D rigid -body spring model to simulate the forces across the articular surfaces was used ${ }^{2}$. By Ellis et al, a 3D pre-surgical planner and an intraoperative guidance system for HTO were developed $^{4}$. Both of them were used for the preoperative planning of HTO. However $2 \mathrm{D}$ rigid-body spring model is $2 \mathrm{D}$ model and $3 \mathrm{D}$ pre-surgical planner is the $3 \mathrm{D}$ model of only around of the knee joint, the alignment of the whole leg in rotation cannot analyze exactly. We developed computer software that calculates the alignment exactly from the 3D whole leg bone models and simulate the operation of HTO. In this study the pre and post-operative alignment of the lower extremities was calculated on 3D models of the whole leg. The differences of FTA and HKA in the rotation of HTO were analyzed. In the case of patient with medial osteoarthritis of the knee, the 
change of HKA in rotation was smaller than that of FTA. FTA was presumed to receive the influence of rotation, in comparison with HKA.

\section{Conclusion}

The rotation of the lower extremity position on the preoperative radiograph influenced the measured value of the axial alignment of the lower extremity. Differences of the HKA were statistically smaller than that of the FTA within the range of preoperative lower extremity rotation on radiographs. In the HTO simulation, errors in cutting proximal tibia bone surface had no influence on the measurement value of the axial alignment of the lower extremities within the range of 10-degree external and internal rotation. However, errors in reattaching proximal bone cutting surface to distal bone surface within the range of 10-degree external and internal rotation had a significant influence on the measurement value of the axial alignment of the lower extremity. Differences of the HKA were statistically smaller than that of the FTA as measured in the HTO simulation of rotational error in cutting the proximal tibia and reattaching bone cutting surface.

\section{References}

1. Bauer GC, et al. Tibial Osteotomy in Gonarthrosis. J. Bone and Joint Surg. 51-A:15451563, 1969.

2. Chao EYS, et al. Computer-aided Preoperative Planning in Knee Osteotomy. Iowa Orthop J. 15:4-18, 1995.

3. Coventry MB. Proximal Tibial Osteotomy. J.Bone and Joint Surg.75-A :196- 201, 1993.

4. Ellis RE, et al. A Surgical Planning and Guidance System for High Tibial Osteotomy. Computer Aided Surgery. 4:264-274, 1999.

5. Hernigou P, et al. Proximal Tibial Osteotomy for Osteoarthritis with Varus Deformity. J. Bone and Joint Surg. 69-A: 332-354, 1987.

6. Insall JN, et al. High Tibial Osteotomy. J.Bone and Joint Surg. 56-A: 1397-1405, 1974.

7. Kettelkamp DB, et al. Results of Proximal Tibial Osteotomy. J.Bone and Joint Surg. 58-A: 952-960, 1976.

8. Krackow KA, et al. A Mathematical Analysys of the Effect of Flexion and Rotation on Apparent Varous/Valgus Alignment at the Knee. Orthopedics; 13:861-868, 1990.

9. Krackow KA, et al. AAOS Instructional 47: 429-436, 1998.

10. 10.Macintosh DL., and Welsh RP. Joint Debridement-A Completement to High Tibial Osteotomy in the Treatment of Degenerative arthritis of the Knee. J. Bone and Joint Surg. 59-A:1094-1097, 1977.

11. Moreland JR ,et al.Radiographic Analysis of the Axial Alignment of the Lower Extremity. J. Bone and Joint Surg. 69-A:745-749, 1987.

12. Rudan JF, et al. High Tibial Osteotomy. Clin.Orthop.268: 157-160, 1990.

13. Swanson KE, et al. Dose Axial limb Rotation Affect the Alignment Measurements in Deformed Limb?. Clin.Orthop:371:246-252, 2000.

14. Wright JG, et al. Measurement of Lower Limb Alignment Using Long Radiographs. J.Bone and Joint Surg.73-B:721-723,1991. 